\title{
Phase Predistortion of a Class-D Outphasing RF Amplifier in 90 nm CMOS
}

\author{
Jonas Fritzin, Ylva Jung, Per Niklas Landin, Peter Handel, \\ Martin Enqvist and Atila Alvandpour
}

\section{Linköping University Post Print}

N.B.: When citing this work, cite the original article.

(C2011 IEEE. Personal use of this material is permitted. However, permission to reprint/republish this material for advertising or promotional purposes or for creating new collective works for resale or redistribution to servers or lists, or to reuse any copyrighted component of this work in other works must be obtained from the IEEE.

Jonas Fritzin, Ylva Jung, Per Niklas Landin, Peter Handel, Martin Enqvist and Atila Alvandpour, Phase Predistortion of a Class-D Outphasing RF Amplifier in $90 \mathrm{~nm}$ CMOS, 2011, IEEE Transactions on Circuits and Systems - II - Express Briefs, (58), 10, 642-646.

http://dx.doi.org/10.1109/TCSII.2011.2164149

Postprint available at: Linköping University Electronic Press

http://urn.kb.se/resolve?urn=urn:nbn:se:liu:diva-71781 


\title{
Phase Predistortion of a Class-D Outphasing RF Amplifier in 90nm CMOS
}

\author{
Jonas Fritzin, Student Member, IEEE, Ylva Jung, Per Niklas Landin, Student Member, IEEE, \\ Peter Händel, Senior Member, IEEE, Martin Enqvist, Member, IEEE, and Atila Alvandpour, Senior Member, IEEE
}

\begin{abstract}
This paper presents a behavioral model structure and a model-based phase-only predistortion method suitable for outphasing RF amplifiers. The predistortion method is based on a model of the amplifier with a constant gain factor and phase rotation for each outphasing signal, and a predistorter with phase rotation only. The method has been used for EDGE and WCDMA signals applied to a Class-D outphasing RF amplifier with an on-chip transformer used for power combining in $90 \mathrm{~nm}$ CMOS. The measured peak power at $2 \mathrm{GHz}$ was $+10.3 \mathrm{dBm}$ with a drain efficiency and power-added efficiency of $39 \%$ and $33 \%$, respectively. For an EDGE 8-PSK signal with a phase error of $3^{\circ}$ between the two input outphasing signals, the measured power at $400 \mathrm{kHz}$ offset was $-65.9 \mathrm{~dB}$ with predistortion, compared to $-53.5 \mathrm{~dB}$ without predistortion. For a WCDMA signal with the same phase error between the input signals, the measured ACLR at $5 \mathrm{MHz}$ offset was $-50.2 \mathrm{dBc}$ with predistortion, compared to $-38.0 \mathrm{dBc}$ without predistortion.

Index Terms-outphasing, CMOS, amplifier, linearization.
\end{abstract}

\section{INTRODUCTION}

$\mathbf{T}$ $\mathrm{O}$ meet the increasing demand for higher data rates, wireless systems target larger bandwidths and higher bandwidth efficiency. For more efficient use of the limited frequency spectrum, non-constant envelope modulation schemes are used, which require high linearity in the transmitter circuits in order to comply with spectral and modulation requirements. To benefit from the CMOS scaling in terms of power consumption and silicon area, a highly "digital" transmitter is desirable in the development of mobile multistandard RF transceivers [1]. Potential solutions are the polar transmitter, shown for EDGE [2], and the outphasing transmitter [3].

In the outphasing transmitter, the original non-constant envelope-modulated signal is used to create two constantenvelope signals, separately amplified by two highly efficient switched amplifiers, like Class-D, and then recombined in a power combiner. In practice the two amplifier stages and signals will experience gain and phase imbalances, creating nonlinearities and spectral distortion [4]. Previous predistortion methods of RF power amplifiers (PA) include model-

Manuscript received February 2, 2011; revised April 29, 2011; accepted July 8,2011 . This work has been supported by the Swedish Foundation for Strategic Research (SSF), the Excellence Center at Linköping-Lund in Information Technology (ELLIIT), the Swedish Research Council (VR), and Ericsson Research, Kista, Sweden. This paper was recommended by Associate Editor Gwee Bah Hwee.

J. Fritzin, Y. Jung, M. Enqvist, and A. Alvandpour are with the Department of Electrical Engineering, Linköping University, SE-581 83 Linköping, Sweden, phone: +46(0)13-282671, e-mail: fritzin@isy.liu.se.

P.N. Landin and P. Händel are with the Signal Processing Lab, ACCESS Linneaus Center, Royal Institute of Technology, Stockholm, Sweden, and with the Center for RF Measurement Technology, University of Gävle, Sweden. P.N. Landin is also with the Dept. ELEC, Vrije Universiteit Brussel, Belgium. based predistorters using model structures such as Volterra series [5], parallel Hammerstein structures [6], or look-up tables [2], which also can be made adaptive [7].

With the increased interest in linearized switched amplifiers, like the outphasing amplifier, suitable amplifier models and predistortion methods are necessary. A number of methods have been presented, but only a few have been verified in measurements. Phase-predistortion was evaluated for Chireix combiners in simulations and by using signal generators in measurements (no PA was used) [4]. A gain/phase imbalanceminimization technique was verified in measurements in [8], and predistortion was used for high power devices in [9]. In [9], the predistorter separately compensates for gain and phase imbalances, where the gain imbalance is eliminated by changing the amplitudes of the input outphasing signals. The gain imbalance can also be eliminated by adjusting the voltage supplies in the output stage [10].

This paper presents a behavioral model structure and a model-based phase-only predistortion method suitable for outphasing RF amplifiers. The predistorter proposed in this paper compensates for both amplitude and phase distortion by changing only the phases of the two input outphasing signals. The proposed predistortion method has been used for EDGE and WCDMA signals applied to a Class-D outphasing RF amplifier with an on-chip transformer used for power combining in 90nm CMOS. The predistortion method is applicable at the baseband level and has not been implemented in hardware.

The outline of the paper is as follows. In Section II, the outphasing concept is explained. In Section III, the behavioral model and the phase predistortion method are described. The implemented amplifier is described in Section IV. In Section V, the measured RF performance and the performance for modulated signals with and without phase-predistortion are presented. In Section VI, the conclusions are provided.

\section{OUtPhasing CONCEPT}

Fig. 1(a) shows the outphasing concept, where a nonconstant envelope-modulated signal

$$
s(t)=r(t) e^{j \alpha(t)}=r_{\max } \cos (\varphi(t)) e^{j \alpha(t)}, 0 \leq r(t) \leq r_{\max }
$$

where $r_{\max }$ is a real-valued constant, is used to create two constant-envelope signals, $s_{1}(t)$ and $s_{2}(t)$, as

$$
\begin{aligned}
& s_{1}(t)=s(t)+e(t)=r_{\max } e^{j \alpha(t)} e^{j \varphi(t)} \\
& s_{2}(t)=s(t)-e(t)=r_{\max } e^{j \alpha(t)} e^{-j \varphi(t)} \\
& e(t)=j s(t) \sqrt{\frac{r_{\max }^{2}}{r^{2}(t)}-1 .}
\end{aligned}
$$


(a)

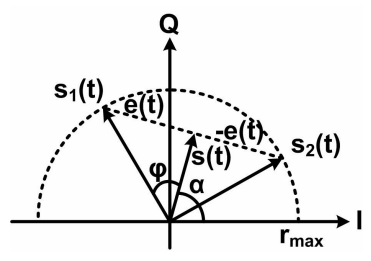

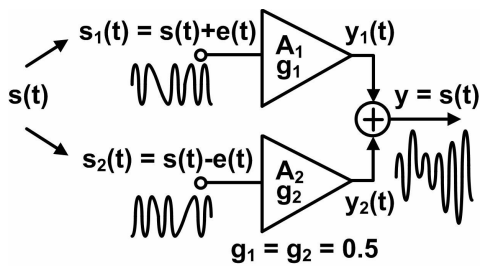

(b)
Fig. 1. (a) Outphasing concept and signal decomposition. (b) Ideal power combining of the two constant-envelope signals.

The two constant-envelope signals contain the original signal, $s(t)$, and a quadrature signal, $e(t)$, and are suitably amplified by switched amplifiers like Class-D. By separately amplifying $s_{1}(t)$ and $s_{2}(t)$, and combining the outputs of the two individual amplifiers, the original signal is reconstructed and amplified as in Fig. 1(b). In the sequel, PA refers to the complete outphasing amplifier and amplifier refers to the switched amplifiers $A_{1}$ and $A_{2}$.

Letting $g_{1}$ and $g_{2}$ denote two real valued gain factors, on $s_{1}(t)$ and $s_{2}(t)$, and $\delta$ a phase mismatch in the path for $s_{1}(t)$, then it is clear from

$$
\begin{aligned}
y(t) & =g_{1} e^{j \delta} s_{1}(t)+g_{2} s_{2}(t) \\
& =\left[g_{1} e^{j \delta}+g_{2}\right] s(t)+\left[g_{1} e^{j \delta}-g_{2}\right] e(t),
\end{aligned}
$$

that besides the amplified signal, a part of the quadrature signal remains. The quadrature signal has a larger bandwidth than the original signal, $s(t)$, and degrades Adjacent Channel Leakage Ratio (ACLR) and reduces the margins to the spectral mask, unless canceled in the power combiner [4]. In order not to allow a residual quadrature component to distort the spectrum or limit the Dynamic Range (DR)

$$
\mathrm{DR}=20 \log _{10}\left(\frac{|\max (y(t))|}{|\min (y(t))|}\right)=20 \log _{10}\left(\frac{\left|g_{1}+g_{2}\right|}{\left|g_{1}-g_{2}\right|}\right)
$$

of the PA, the phase and gain mismatches between $s_{1}(t)$ and $s_{2}(t)$ must be minimized [4]. The DR sets limits on which output amplitudes can be achieved with the PA, but within the DR the PA can achieve all amplitudes by changing the phases of the outphasing signals. The constant gain, $g_{1}$ and $g_{2}$, approximations are especially suitable for Class-D amplifiers, where the output can be considered as an ideal voltage source whose output voltage is independent of the load [11]. This also makes Class-D suitable for non-isolating combiners like transformers, recently demonstrated in [12].

\section{PREDISTORTION}

A digital predistorter (DPD) has been designed to predistort the input of the implemented PA to cancel the nonlinearities, and is ideally the inverse of the PA transfer function.

Models describing the behavior between the PA input and output as well as the PA inverse, the DPD, have been estimated using estimation data sets (EDGE and WCDMA signals). The DPD was applied to a second data set, validation input data, and the DPD output was applied to the PA. The DPD was estimated in cascade with the PA model, as in Fig. 2(c), to assure that the pre-inverse is obtained, which is not necessarily the same as the post-inverse in the general case [13]. Moreover,

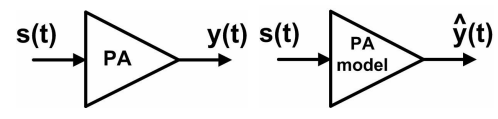

(a)

(b)

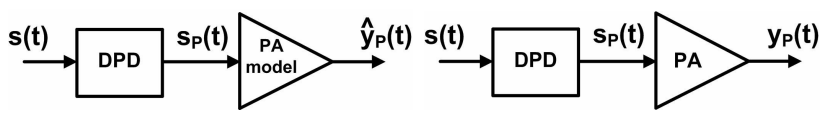

(c)

(d)

Fig. 2. The inputs and outputs of (a) the PA, (b) the PA model, (c) the DPD and PA model (d) the DPD and PA. (c) illustrates the DPD estimation setup and (d) the intended use of the DPD.

most system identification methods assume additive noise on the output [14], whereas an estimation of the post-inverse, from the output $y(t)$ to the input $s(t)$, would have the noise at the estimation input signal $y(t)$.

The largest amplitude of the input signal and the measured output signal were normalized to 1 , thus $g_{1}+g_{2}$ was normalized to 1 during modeling. Despite the fact that the PA is analog and the baseband model is time-discrete, the notation $t$ is used for indicating the dependency of time. Based on the context, $t$ may thus be a continuous or discrete quantity.

\section{A. PA model}

The PA model was estimated from the input $s(t)$ and the measured output $y(t)$ of the PA as in Fig. 2(a). With the gain mismatch between $g_{1}$ and $g_{2}$ and a time delay $\tau$, a first model structure, Model structure A, was suggested as

$$
y_{A}(t)=g_{1} s_{1}(t+\tau)+g_{2} s_{2}(t),
$$

where $\tau$ is a real valued constant. Applying this model to estimation input data, the phase error appeared to be dependent on the amplitude of the input; the phase shift increases with an increasing input amplitude. Fig. 1(a) shows that the amplitude information of the original input signal $s(t)$ can be found in the angle between $s_{1}(t)$ and $s_{2}(t)$,

$$
\Delta_{\psi}\left(s_{1}, s_{2}\right)=\arg \left(s_{1}(t)\right)-\arg \left(s_{2}(t)\right),
$$

where $\Delta_{\psi}=2 \varphi$ in Fig. 1(a). Here, the phases are assumed to be unwrapped.

To model the amplitude dependent phase shift without changing the constant amplitude of the signals $s_{1}(t)$ and $s_{2}(t)$, a model structure with an exponential function with a polynomial of order $n$ in the exponent was used. This model, referred to as Model structure B, can be described by

$$
\begin{aligned}
y_{B}(t)= & g_{1} s_{1}(t) e^{j p\left(\eta_{1}, \Delta_{\psi}\left(s_{1}, s_{2}\right)\right)} \\
& +g_{2} s_{2}(t) e^{j p\left(\eta_{2}, \Delta_{\psi}\left(s_{1}, s_{2}\right)\right)}
\end{aligned}
$$

where $\eta_{1}, \eta_{2}$ are the vectors of polynomial coefficients in

$$
p\left(\eta_{k}, \Delta_{\psi}\left(s_{1}, s_{2}\right)\right)=\sum_{i=0}^{n} \eta_{k, i} \Delta_{\psi}\left(s_{1}, s_{2}\right)^{i}, \quad k=1,2 .
$$

Model structure $\mathrm{B}$ with the additional constraint $\eta_{1, i}=$ $\eta_{2, i}, i=1,2, \ldots, n$ is referred to as Model structure C.

The model parameters in a given model structure are estimated by minimizing a quadratic cost function [14] as in

$$
\hat{\theta}=\underset{\theta}{\operatorname{argmin}} \sum_{t=1}^{N}|y(t)-\hat{y}(t, \theta)|^{2}
$$




$$
\begin{aligned}
\hat{y}(t, \theta)= & g_{1} s_{1}(t) e^{j p\left(\eta_{1}, \Delta_{\psi}\left(s_{1}, s_{2}\right)\right)} \\
& +g_{2} s_{2}(t) e^{j p\left(\eta_{2}, \Delta_{\psi}\left(s_{1}, s_{2}\right)\right)}
\end{aligned}
$$

where $\theta=\left[\begin{array}{llll}g_{1} & g_{2} & \eta_{1}^{T} & \eta_{2}^{T}\end{array}\right]^{T} \in \mathbb{R}^{2 n+4}, y(t)$ is the measured output data and $\hat{y}(t)$ is the modeled output, compare Fig. 2(a) and 2(b). This structure leads to a nonlinear and possibly nonconvex optimization problem, so the minimization algorithm might find a local optimum instead of a global.

\section{B. DPD model}

When identifying the DPD model, the model structure was assumed to be the same as for the PA model, motivated by the Stone-Weierstrass theorem (Theorem 7.26 [15]). The minimization criterion used was

$$
\begin{array}{r}
\hat{\theta}_{\mathrm{DPD}}=\underset{\theta_{\mathrm{DPD}}}{\operatorname{argmin}} \sum_{t=1}^{N}\left|s(t)-\hat{y}_{\mathrm{P}}\left(t, \theta_{\mathrm{DPD}}\right)\right|^{2}, \\
\hat{y}_{\mathrm{P}}\left(t, \theta_{\mathrm{DPD}}\right)= \\
\hat{g}_{1} s_{1, \mathrm{P}}(t) e^{j p\left(\hat{\eta}_{1}, \Delta_{\psi}\left(s_{1, \mathrm{P}}, s_{2, \mathrm{P}}\right)\right)} \\
+\hat{g}_{2} s_{2, \mathrm{P}}(t) e^{j p\left(\hat{\eta}_{2}, \Delta_{\psi}\left(s_{1, \mathrm{P}}, s_{2, \mathrm{P}}\right)\right)}
\end{array}
$$

where

$$
s_{k, \mathrm{P}}(t)=s_{k}(t) e^{j p\left(\eta_{k, \mathrm{DPD}}, \Delta_{\psi}\left(s_{1}, s_{2}\right)\right)}, \quad k=1,2,
$$

and $\theta_{\mathrm{DPD}}=\left[\begin{array}{ll}\eta_{1, \mathrm{DPD}}^{T} & \eta_{2, \mathrm{DPD}}^{T}\end{array}\right]^{T} \in \mathbb{R}^{2 n+2}$. The signal $\hat{y}_{p}(t)$ is the output from the PA model, using a predistorted input, as in Fig. 2(c). The resulting estimated parameter vector $\hat{\theta}_{\mathrm{DPD}}$ contains the DPD model parameters. With these parameters the signals $s_{1, \text { val, } \mathrm{P}}(t)$ and $s_{2, \text { val, } \mathrm{P}}(t)$ were created using the validation data and applied to the PA as in Fig. 2(d). The measured results are presented in Section V.

\section{Theoretical motivation of the DPD Model}

At the output of the combiner, the perfect predistorter should lead to a (normalized) output which is a copy of the input, i.e. no amplitude or phase alteration should occur. This holds if the signals $y_{1}(t), y_{2}(t)$ from Fig. 1 are equal to $s_{1}(t), s_{2}(t)$ from (2) except for the gain. Nonidentical gain factors result in a scaling and an added phase shift, and $y_{k}(t)$ should instead be compared to $\tilde{s}_{k}(t)$ (the input signal $s(t)$ decomposed as $y(t)$ with gain factors $g_{1}$ and $\left.g_{2}\right)$, so that $\tilde{s}_{1}(t)+\tilde{s}_{2}(t)=$ $s(t),\left|\tilde{s}_{k}(t)\right|=g_{k}, k=1,2$ and $\arg \left(\tilde{s}_{1}(t)\right) \geq \arg \left(\tilde{s}_{2}(t)\right)$. The phase shifts $\xi_{k}=\arg \left(\tilde{s}_{k}(t)\right)-\arg \left(s_{k}(t)\right), k=1,2$, depend only on $g_{1}$ and $g_{2}$ and $|s(t)|$, or $\Delta_{\psi}$, where $\Delta_{\psi}=\Delta_{\psi}\left(s_{1}, s_{2}\right)$.

Let $f_{k}(v)$ be the (perfect) model of the phase shift in the PA defined as

$$
\begin{aligned}
y(t) & =y_{1}(t)+y_{2}(t) \\
& =g_{1} s_{1}(t) e^{j f_{1}\left(\Delta_{\psi}\right)}+g_{2} s_{2}(t) e^{j f_{2}\left(\Delta_{\psi}\right)}
\end{aligned}
$$

and $h_{k}(v)$ be the (ideal) predistorter working on input $s_{k}(t)$,

$$
\begin{aligned}
y_{\mathrm{P}}(t) & =g_{1} s_{1, \mathrm{P}}(t) e^{j f_{1}\left(\Delta_{\psi}\left(s_{1, \mathrm{P}}, s_{2, \mathrm{P}}\right)\right)}+g_{2} s_{2, \mathrm{P}}(t) e^{j f_{2}\left(\Delta_{\psi}\left(s_{1, \mathrm{P}}, s_{2, \mathrm{P}}\right)\right)} \\
s_{k, \mathrm{P}}(t) & =s_{k}(t) e^{j h_{k}\left(\Delta_{\psi}\left(s_{1}, s_{2}\right)\right)}, \quad k=1,2
\end{aligned}
$$

Defining $\tilde{f}(v)=v+f_{1}(v)-f_{2}(v)$ and $\tilde{h}(v)=v+h_{1}(v)-$ $h_{2}(v)$, and requiring no output amplitude change leads to

$$
\begin{aligned}
\Delta_{\psi}\left(y_{1, \mathrm{P}}, y_{2, \mathrm{P}}\right) & =\tilde{f}\left(\tilde{h}\left(\Delta_{\psi}\right)\right)=\xi\left(\Delta_{\psi}\right) \\
\tilde{h}\left(\Delta_{\psi}\right) & =\tilde{f}^{-1}\left(\xi\left(\Delta_{\psi}\right)\right),
\end{aligned}
$$

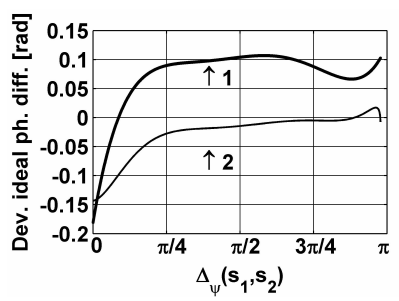

(a)

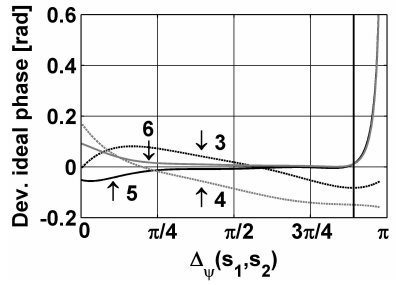

(b)
Fig. 3. (a) The deviation from the ideal phase difference at the output without predistortion (line 1) and with predistortion (line 2), for Model structure B identified for EDGE input. (b) The deviations from the ideal phase of the signals $y_{1}$ and $y_{2}$ without predistortion ( $y_{1}$ line 3 and $y_{2}$ line 4) and with predistortion ( $y_{1}$ line 5 and $y_{2}$ line 6 , lines 5 and 6 reach a value of 1.06). The measurements with $\Delta_{\psi}>2.8$ (right of the vertical line) represent $0.6 \%$ of the data.

where $\xi\left(\Delta_{\psi}\right)=\Delta_{\psi}+\xi_{1}\left(\Delta_{\psi}\right)-\xi_{2}\left(\Delta_{\psi}\right)$.

The phase change can be calculated as

$$
\arg \left(y_{1, \mathrm{P}}(t)\right)=\arg \left(s_{1}(t)\right)+h_{1}\left(\Delta_{\psi}\right)+f_{1}\left(\tilde{h}\left(\Delta_{\psi}\right)\right)
$$

and requiring $\arg \left(y_{1, \mathrm{P}}(t)\right)=\arg \left(\tilde{s}_{1}(t)\right)=\arg \left(s_{1}\right)+\xi_{1}\left(\Delta_{\psi}\right)$ leads to

$$
h_{1}\left(\Delta_{\psi}\right)=-f_{1}\left(\tilde{h}\left(\Delta_{\psi}\right)\right)+\xi_{1}\left(\Delta_{\psi}\right)
$$

if no phase shift is to occur, and analogously

$$
h_{2}\left(\Delta_{\psi}\right)=-f_{2}\left(\tilde{h}\left(\Delta_{\psi}\right)\right)+\xi_{2}\left(\Delta_{\psi}\right) .
$$

Choosing predistorters according to (18) and (19) used as in (15), it is possible to achieve a perfect compensation in the PA described by (14). Two independent predistorters are used, one for each signal $s_{1}(t)$ and $s_{2}(t)$. Though the predistorters used are not ideal but estimated using measured data, the same requirements of no phase shift and no amplitude change are valid. The resulting deviation from the ideal phase difference leads to a change in output amplitude with and without predistortion for EDGE as shown in Fig. 3(a), for Model structure B, and is clearly reduced by the DPD. The deviations from the ideal phase in each signal path should be zero and the DPD reduces the deviations in a large part of the working area, as seen in Fig. 3(b). The output phase deviation is also improved, shown by the small deviation from the ideal phases of $y_{1}(t)$ and $y_{2}(t)$ after predistortion.

\section{IMPLEMENTATION OF THE Class-D OUTPHASING RF AMPLIFIER}

Fig. 4 shows the implemented Class-D outphasing amplifier with an inverter-based output stage and an on-chip transformer as power combiner. Fig. 5 shows the chip photo. The chip was bonded on a FR4 printed circuit board and connected with bond-wires. The NMOS, $\mathrm{T}_{1}$ and $\mathrm{T}_{3}$, and PMOS, $\mathrm{T}_{2}$ and $\mathrm{T}_{4}$, transistor widths in the output stage were $60 \mu \mathrm{m}$ and $180 \mu \mathrm{m}$, respectively. An off-chip capacitor $\left(C_{\text {tune }}\right)$ was used to set the frequency characteristics, optimized at $2 \mathrm{GHz}$. The buffers of the output stage were tapered buffers with tapering factor $\lambda=3$. A $4: 3$ turns ratio was used in the transformer for a high coupling factor and a high bandwidth, but constrains the output power to levels suitable for transceivers and low-power PAs. The self-inductances of the galvanically isolated primary, 


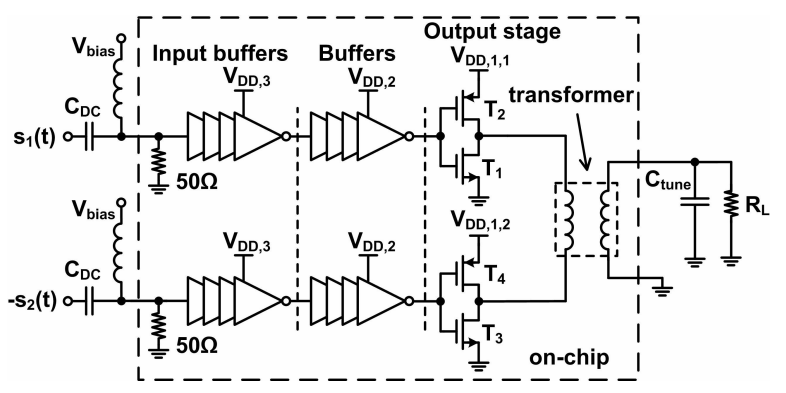

Fig. 4. Implemented outphasing amplifier with inverters in the output stage.

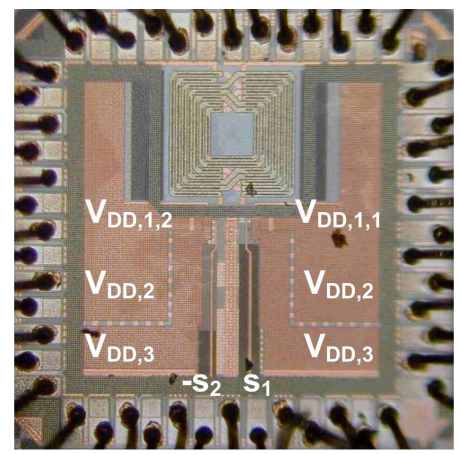

Fig. 5. Photo of the chip with size $1 \times 1 \mathrm{~mm}^{2}$.

$L_{\mathrm{p}}$, and secondary, $L_{\mathrm{s}}$, windings were $3.7 \mathrm{nH}$ and $2.5 \mathrm{nH}$, respectively. The windings were implemented in the top three metal layers and had a total thickness of $2 \mu \mathrm{m}$. The quality factors, $Q_{\mathrm{p}}$ and $Q_{\mathrm{s}}$, were 10 and 8 at $2 \mathrm{GHz}$, respectively. Onchip resistors were used for an equivalent input impedance of $50 \Omega$ at the chip edge for each RF input $\left(s_{1}(t)\right.$ and $\left.-s_{2}(t)\right)$.

\section{Measurement Results}

\section{A. Measured RF Performance}

Fig. 6 shows the measured maximum output power $\left(P_{\text {out }}\right)$ with the drain efficiency (DE) and power-added efficiency (PAE) over frequency for the amplifier only (the predistortion method has not been implemented in hardware). $V_{\mathrm{DD}}$ and $V_{\text {bias }}$ were $1.3 \mathrm{~V}$ and $0.65 \mathrm{~V}$, respectively. The $3 \mathrm{~dB}$ bandwidth was $2 \mathrm{GHz}(1-3 \mathrm{GHz})$. At $2 \mathrm{GHz}$, the output power was $+10.3 \mathrm{dBm}$ with a DE and PAE of $39 \%$ and $33 \%$, respectively. The gain was $23 \mathrm{~dB}$ from the buffers to the output. The minimum and maximum output power and DR of the PA are plotted in Fig. 7, where $P_{\text {out,max }}=P_{\text {out }}$ in Fig. 6 . In simulations at $2 \mathrm{GHz}$, the DR was $\sim 30 \mathrm{~dB}$ for a large number of load impedances $\left(R_{L}\right)$. Thus, the predistortion method is expected to give similar ACLR performance as reported in Section V-B even if the load is changed.

\section{B. Measured Performance of Modulated Signals}

The Peak-to-Average Power Ratios (PAPR) of the EDGE and WCDMA signals were $3.0 \mathrm{~dB}$ and $3.2 \mathrm{~dB}$, respectively. The spectrum of the estimation data sets are shown in Fig. 8(d) and Fig. 9(d). The signal generator was an SMU200A with two phase-coherent RF outputs and an arbitrary waveform generator where $s_{1}(t)$ and $s_{2}(t)$ were stored. For the computation of the model parameters a variety of algorithms are available to solve the nonlinear optimization problem. In this paper, the Matlab routine fminsearch, based on the Nelder-Mead simplex

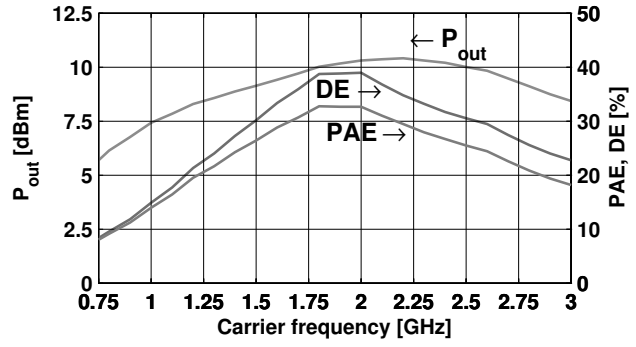

Fig. 6. Measured output power $\left(P_{\text {out }}\right)$, DE and PAE over frequency.

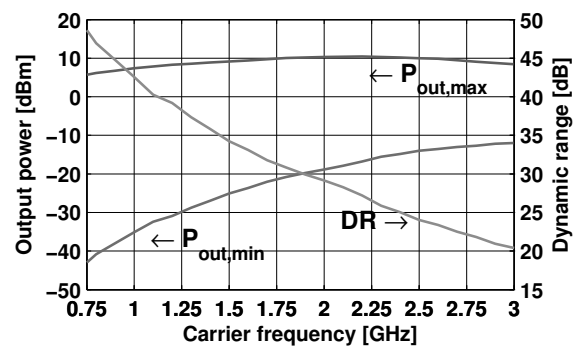

Fig. 7. Measured maximum output power, $P_{\text {out,max }}$, and minimum output power, $P_{\text {out,min }}$, and dynamic range, DR, over frequency.

method, was used. The estimation and validation data sets contain $N_{\text {id }}$ and $N_{\text {val }}$ samples, respectively. The input and output sampling frequencies are denoted $f_{\mathrm{s}}$ and $f_{\mathrm{s}, \text { out }}$, respectively. To minimize the influence of measurement noise, the signals were measured $K$ times, and a mean was calculated. The data collection parameters are shown in Table I.

Measurements with two amplitude-matched signal generators, i.e. $g_{1}=g_{2}=0.5$, show that phase errors of $12^{\circ}$ and $4^{\circ}$ are acceptable for WCDMA and EDGE to meet the ACLR and $400 / 600 \mathrm{kHz}$ offset requirements. Thus, a predistortion implementation would require a phase resolution of at least 7 bits, i.e. $360^{\circ} / 2^{7}=2.81^{\circ}$. For each bit of increased phase resolution, the ACLR and margins to the spectral mask improve by $\sim 3 \mathrm{~dB}$.

The measured performance of the amplifier for modulated signals are summarized in Table II and Table III. For the EDGE signal at $1 \mathrm{GHz}$, the phase offset between $s_{1}(t)$ and $s_{2}(t)$ in the baseband was adjusted to minimize phase mismatch (ideally $180^{\circ}$ between the two RF inputs for nonmodulated $s_{1}(t)$ and $-s_{2}(t)$ in Fig. 4, i.e. maximum output power for a continuous signal). The margins to the spectral mask were 4.0 and $7.0 \mathrm{~dB}$ at 400 and $600 \mathrm{kHz}$ offset from the carrier, and no predistortion was applied. At $2 \mathrm{GHz}$, including phase adjustments, the margins to the mask have disappeared as shown in Fig. 8(a). As the phase error cannot be assumed to be $0^{\circ}$ in a transceiver, a phase error of $3^{\circ}$ was added and led to a violated spectral mask as in Fig. 8(b).

The estimation output data $y(t)$ were used in the predistortion method to extract the model parameters, using

TABLE I

DATA COLLECTION

\begin{tabular}{lccccc}
\hline & $N_{\mathrm{id}}$ & $N_{\text {val }}$ & $f_{\mathrm{s}}$ & $f_{\mathrm{s}, \text { out }}$ & $K$ \\
\hline EDGE & 40001 & 80001 & $8.67 \mathrm{MHz}$ & $34.68 \mathrm{MHz}$ & 150 \\
\hline WCDMA & 153600 & 153600 & $61.44 \mathrm{MHz}$ & $61.44 \mathrm{MHz}$ & 200 \\
\hline
\end{tabular}




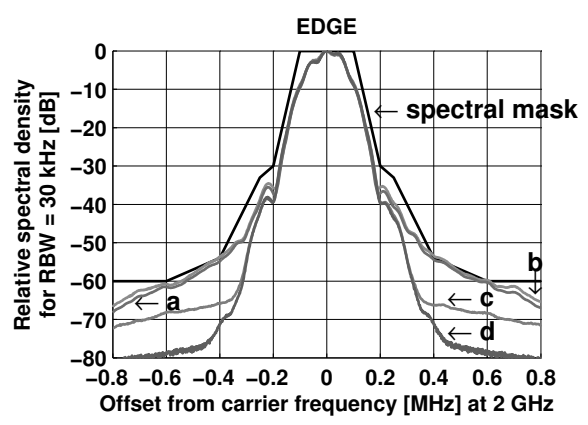

Fig. 8. Measured EDGE output spectrum at $2 \mathrm{GHz}$.

(a) Without phase error between $s_{1}(t)$ and $s_{2}(t)$.

(b) With $3^{\circ}$ phase error between $s_{1}(t)$ and $s_{2}(t)$.

(c) When DPD is applied to (b).

(d) Spectrum of estimation signal. Spectrum of validation signal was similar.

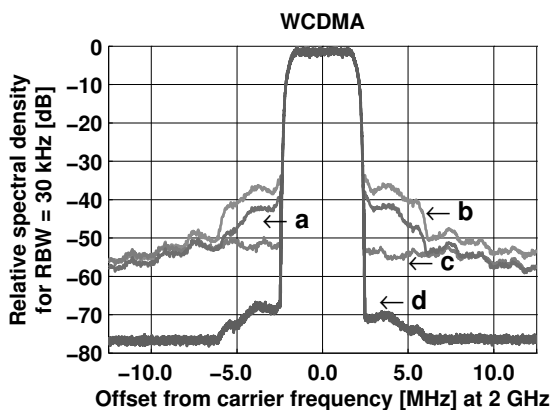

Fig. 9. Measured WCDMA spectrum at $2 \mathrm{GHz}$. (a)-(d) as in Fig. 8.

Model structure B with $n=5$. The predistorted input signals, $s_{1, \mathrm{val}, \mathrm{P}}(t)$ and $s_{2, \mathrm{val}, \mathrm{P}}(t)$, were computed for the validation input signal, resulting in an output spectrum as shown in Fig. 8(c). The measured power at 400 and $600 \mathrm{kHz}$ offsets were -65.9 and $-68.2 \mathrm{~dB}$, respectively. The average power at $2 \mathrm{GHz}$ was $+7 \mathrm{dBm}$ with $22 \%$ PAE and RMS EVM of $2 \%$.

Fig. 9(a) shows the measured WCDMA spectrum at $2 \mathrm{GHz}$, with minimized phase mismatch and no predistortion. Adding $3^{\circ}$ of phase error, a distorted spectrum as in Fig. 9(b) was measured. The phase predistortion method, using Model Structure $\mathrm{C}$ with $n=4$, for the validation signal, improves the measured ACLR at $5 \mathrm{MHz}$ offset to $-50.2 \mathrm{dBc}$, with a spectrum shown in Fig. 9(c). At $1 \mathrm{GHz}$, similar performance was achieved as seen in Table III. The channel power at $2 \mathrm{GHz}$ was $+6.3 \mathrm{dBm}$ with PAE of $22 \%$ and RMS composite EVM of $1.4 \%(0.6 \%$ after DPD). The RX noise floor after predistortion, assuming a $45 \mathrm{MHz}$ offset, was $-140 \mathrm{dBc} / \mathrm{Hz}$ and limited by the signal generator phase noise, not the outphasing amplifier. Before predistortion, the noise floor was $-138 \mathrm{dBc} / \mathrm{Hz}$.

The measured spectral performance at $400 \mathrm{kHz}$ offset and the ACLR at $5 \mathrm{MHz}$ is comparable to state-of-the-art EDGE [2] and WCDMA [16] transmitters.

\section{Conclusions}

This paper presents a behavioral model structure and a model-based phase-only predistortion method suitable for outphasing RF amplifiers. The predistortion method is applicable at the signal generation level in the baseband, and it has been used for EDGE and WCDMA signals applied to a Class-D outphasing RF amplifier with an on-chip transformer for power combining in $90 \mathrm{~nm}$ CMOS. In measurements at $2 \mathrm{GHz}$, the
TABLE II

MEASURED SPECTRAL PERFORMANCE OF THE EDGE SIGNAL

\begin{tabular}{llllll}
\hline Freq. & Freq. offset & Spec. & Meas. (a) & Meas. (b) & Meas. (c) \\
\hline $2 \mathrm{GHz}$ & $400 \mathrm{kHz}$ & $-54 \mathrm{~dB}$ & $-54.4 \mathrm{~dB}$ & $-53.5 \mathrm{~dB}$ & $-65.9 \mathrm{~dB}$ \\
\hline & $600 \mathrm{kHz}$ & $-60 \mathrm{~dB}$ & $-60.3 \mathrm{~dB}$ & $-59.9 \mathrm{~dB}$ & $-68.2 \mathrm{~dB}$ \\
\hline
\end{tabular}

(a) With no phase error and no DPD.

(b) For a $3^{\circ}$ phase error and no DPD.

(c) When DPD is applied to (b).

TABLE III

MEASURED SPECTRAL PERFORMANCE OF THE WCDMA SIGNAL

\begin{tabular}{llllll}
\hline Freq. & ACLR & Spec. & Meas. (a) & Meas. (b) & Meas. (c) \\
\hline $1 \mathrm{GHz}$ & $5 \mathrm{MHz}$ & $-33 \mathrm{dBc}$ & $-40.6 \mathrm{dBc}$ & $-39.4 \mathrm{dBc}$ & $-53.6 \mathrm{dBc}$ \\
\hline & $10 \mathrm{MHz}$ & $-43 \mathrm{dBc}$ & $-59.8 \mathrm{dBc}$ & $-56.2 \mathrm{dBc}$ & $-60.3 \mathrm{dBc}$ \\
\hline $2 \mathrm{GHz}$ & $5 \mathrm{MHz}$ & $-33 \mathrm{dBc}$ & $-43.4 \mathrm{dBc}$ & $-38.0 \mathrm{dBc}$ & $-50.2 \mathrm{dBc}$ \\
\hline & $10 \mathrm{MHz}$ & $-43 \mathrm{dBc}$ & $-53.9 \mathrm{dBc}$ & $-50.9 \mathrm{dBc}$ & $-52.2 \mathrm{dBc}$
\end{tabular}

See Table II for description of (a)-(c).

DPD proved to be successful and improved the margin to the EDGE spectral mask at $400 \mathrm{kHz}$ and the WCDMA ACLR at $5 \mathrm{MHz}$ offset by 12.2-12.4 dB.

\section{REFERENCES}

[1] X. He and J. van Sinderen, "A 45nm WCDMA Transmitter Using Direct Quadrature Voltage Modulator with High Oversampling Digital FrontEnd," in ISSCC Dig. Tech. Papers, Feb. 2010, pp. 62-63.

[2] J. Mehta, V. Zoicas, O. Eliezer, R. Staszewski, S. Rezeq, M. Entezari, and P. Bolsara, "An Efficient Linearization Scheme for a Digital Polar EDGE Transmitter," IEEE Transactions on Circuits and Systems II Express Briefs, vol. 57, no. 3, pp. 193-197, Mar. 2010.

[3] D. Cox, "Linear Amplification with Nonlinear Components," IEEE Trans. Commun., vol. COM-23, pp. 1942-1945, Dec. 1974.

[4] A. Birafane and A. Kouki, "Phase-Only Predistortion for LINC Amplifiers With Chireix-Outphasing Combiners," IEEE Trans. Microw. Theory Techn., vol. 53, no. 6, pp. 2240-2250, Jun. 2005.

[5] A. Zhu, P. Draxler, J. Yan, T. Brazil, D. Kimball, and P. Asbeck, "OpenLoop Digital Predistorter for RF Power Amplifiers Using Dynamic Deviation Reduction-Based Volterra Series," IEEE Trans. Microw. Theory Techn., vol. 56, no. 7, pp. 1524-1534, Jul. 2008.

[6] L. Anttila, P. Händel, and M. Valkama, "Joint Mitigation of Power Amplifier and I/Q Modulator Impairments in Broadband Direct-Conversion Transmitters," IEEE Trans. Microw. Theory Techn., vol. 58, no. 4, pp. 730-739, Apr. 2010.

[7] Y. Woo, J. Kim, J. Yi, S. Hing, I. Kim, J. Moon, and B. Kim, "Adaptive Digital Feedback Predistortion Technique for Linearizing Power Amplifiers," IEEE Trans. Microw. Theory Techn., vol. 55, no. 5, pp. 932-940, May 2007.

[8] X. Zhang, L. Larson, P. Asbeck, and P. Nanawa, "Gain/Phase ImbalanceMinimization Techniques for LINC Transmitters," IEEE Trans. Microw. Theory Techn., vol. 49, no. 12, pp. 2507-2516, Jun. 2001.

[9] A. Huttunen and R. Kaunisto, "A 20-W Chireix Outphasing Transmitter for WCDMA Base Stations," IEEE Trans. Microw. Theory Techn., vol. 55, no. 12, pp. 2709-2718, Dec. 2007.

[10] I. Hakala, D. Choi, L. Gharavi, N. Kajakine, J. Koskela, and R. Kaunisto, "A 2.14-GHz Chireix Outphasing Transmitter," IEEE Trans. Microw. Theory Techn., vol. 53, no. 6, pp. 2129-2138, Jun. 2005.

[11] J. Yao and S. Long, "Power Amplifier Selection for LINC Application," IEEE Trans. Circuits Syst. II, Exp. Briefs, vol. 53, no. 8, pp. 763-766, Aug. 2006.

[12] H. Xu, Y. Palaskas, A. Ravi, and K. Soumyanath, "A Highly Linear 25dBm Outphasing Power Amplifier in 32nm CMOS for WLAN Application," in IEEE Europ. Solid-State Circ. Conf., Sep. 2010, pp. 306-309.

[13] P. Kenington, High-Linearity RF Amplifier Design. Artech House, 2000.

[14] L. Ljung, System Identification, Theory for the user, 2nd ed. Prentice Hall PTR, 1999.

[15] W. Rudin, Principles of Mathematical Analysis, 3rd ed. McGraw-Hill Book Co., 1976.

[16] Q. Huang, J. Rogin, X. Chen, D. Tschopp, T. Burger, T. Christen, D. Papadopolous, I. Kouchev, C. Martelli, and T. Dellsperger, "A TriBand SAW-Less WCDMA/HSPA RF CMOS Transceiver, with On-Chip DC-DC Converter Connectable to Battery," in ISSCC Dig. Tech. Papers, Feb. 2010, pp. 60-61. 